\title{
Dorsal vein thrombosis of the penis presenting to an STD clinic
}

\author{
Derek T P Evans, Olga E R Ward
}

\begin{abstract}
Objective-To describe the clinical assessment, diagnosis and differential diagnosis of dorsal vein thrombosis of the penis (DVTP) and to observe its natural course over time.
\end{abstract}

Design-A descriptive study of six patients presenting with penile swelling to an STD clinic over a twenty month period.

Subjects-Six male patients between the ages of 22 and 46 years who self-presented to an STD clinic in Perth, Western Australia during a period from October 1991 to June 1993.

Methods-Initial history, examination and follow up were undertaken as routine for all STD clinic patients. This was supplemented with later exhaustive history taking; full cardiovascular, fundoscopic, abdominal and genital examination; blood screening for coagulation defects, glucose level, autoantibodies, ESR, urea, electrolytes, calcium, creatinine and liver function test; and duplex doppler ultrasound scanning.

Results-No consistent abnormalities were detected on clinical examination, nor on blood testing. Ultrasound revealed one case of rupture of the corpus cavernosum, with haematoma and thrombus formation. There were two cases of pure DVTP demonstrable with ultrasound and two cases in which spontaneous resolution of clinical DVTP has occurred. The sixth patient declined further investigation and followup, but also displayed the clinical features of DVTP. Coagulation abnormalities as seen in elevated antithrombin III levels are of unknown significance.

Conclusions-DVTP and ruptured corpus cavernosum should be considered in the differential diagnosis of gradual onset penile swelling and/or deformity. Its natural course tends to be one of spontaneous resolution. No sexual or urinary symptoms or dysfunction were experienced, even in the presence of persistent thrombus. Directed and specific investigation only, depending on the clinical state of the patient, should be carried out.

(Genitourin Med 1994;70:406-409)

Introduction

Swelling of the penis is often attributed to trauma during sexual activity. Whilst this may often be the case, we wish to describe several cases of more insidious onset, where the links with sexual trauma are unclear. As well as DVTP, rupture of the corpus cavernosum (RCC) may also present with relatively minor swelling and slow onset, particularly if the leaking area has rapidly sealed off. The two conditions of DVTP and RCC may coexist.

The entity of "penile fracture" implies a disruption of the tunica albuginea. Such fractures frequently occur during vigorous intercourse, with the patient often reporting a distinct snapping feeling and sound. Immediate onset of swelling and pain occurs and Buck's fascia is usually disrupted. It is not unusual for a fracture to also involve the corpus spongiosum and retrograde urethrogram is part of the routine evaluation of the suspected fractured penis. ${ }^{1}$ With uncomplicated rupture of the corpus spongiosum, there are good results with either conservative management or immediate repair. With rupture of the corpus cavernosum, however, severe chordee can develop. ${ }^{1}$ Early exploration with evacuation of haematoma, debridement and repair is indicated. If the urethra is involved, immediate repair is mandatory. ${ }^{1}$

Fracture of the penis is an injury occurring only during erection, involving one or both corpora cavernosa and, in about $30 \%$ of cases, partial or complete urethral rupture. ${ }^{2}$ In the flaccid state, the tunica albuginea is $2 \mathrm{~mm}$ thick, while during erection it reduces to 0.5 $\mathrm{mm}$; making the erect penis much more vulnerable to trauma. Bleeding as a result of fracture takes place in all fascial compartments and may be compounded with urinoma in urethral rupture.

Penile fracture is not an uncommon urologic injury. ${ }^{35}$ All our patients associated their symptoms with sexual activity, rather than with the self-mutilation or kicking injuries to the perineum as reported by Gallagher and Kiser. ${ }^{4}$ Conservative treatment is now rarely used owing to a significant incidence of impotence and curvature distal to the fracture. ${ }^{6}$ Fernstrom ${ }^{7}$ was the first author to suggest a surgical approach. Urethroplasty may be necessary. ${ }^{5}$ We were unable to find any reference to DVTP in relation to sexual trauma in the literature.

\section{Methods}

Six patients presenting with penile swelling to the STD clinic in Perth between October
Murray Street Clinic, Perth, Western

Australia

D T P Evans 
1991 and June 1993 were evaluated. A history and genital examination were performed at presentation, as for all STD clinic patients. Detailed medical, sexual, family and social histories and repeat examination of the genitalia and vascular system were taken at final followup (September 1993 in all cases). Also performed at final followup were coagulation studies, liver function tests, autoantibodies, blood sugar, ESR, urea, electrolytes, calcium and creatinine and duplex colour flow scans with doppler ultrasonography. Patient F declined followup.

\section{Results}

A. A 41 year old unemployed man presented on 7 April 1993 with lumps appearing in the penis over a two week period. There was evidence of haematoma formation. Examination revealed a $4 \mathrm{~cm}$ linear thrombus in the dorsal vein at the mid-shaft. The penis bent to the left and ventrally on erection. An initial diagnosis of Peyronie's disease was made. Subsequent doppler scanning refuted this. Duplex Doppler colour flow ultrasound results were:- Corpora are normal. $9 \mathrm{~mm}$ area of superficial thickening on dorsum of shaft. Unable to demonstrate any flow in dorsal vein. Probable DVTP present. Protein S functional was 156 (65-140\%) Antithrombin III was $153 \%(82-105 \%)$ and protein $C$ antigenic 146 (70-140\%). Final diagnosis: DVTP

B. A 45 year old electrician presented on 22 June 1993 with a swollen distal penile shaft and a hemicircumferential lump at mid-shaft. He was a type 2 diabetic controlled on diet alone. He was referred for urological assessment and managed conservatively. Duplex Doppler results were:- Normal flow in deep dorsal vein. Normal anatomy. No evidence of any thrombosis. Antithrombin III was $108 \%$ and protein $C$ antigenic was 147. Final diagnosis: resolved DVTP.

C. A 46 year old orderly presented on 9 October 1991. Examination revealed a $5 \mathrm{~cm}$ hard cordlike structure in the dorsal vein of the penis, with some associated lymphoedema. By 18 October 1991 the dorsal vein had softened considerably. A chest radiograph, colonoscopy and sigmoidoscopy to rule out malignancy were all normal. Duplex Doppler results were:- Normal anatomy. No evidence of fibrosis in the intercorporal septa. Normal flow demonstrated on colour flow mapping of the deep and superficial veins of the penis. Antithrombin III was $120 \%$. Final diagnosis: resolved DVTP.

D. A 29 year old computer operator presented on 4 March 1993. A lump had developed in the penis during sexual intercourse two weeks prior to presentation, with haematospermia. Limited coagulation profile at the time was within normal limits. There was a palpable lump, $1 \mathrm{~cm}$ in diameter, dorsally at the root of the penis and a $3 \mathrm{~cm} \times 2$ $\mathrm{cm}$ lump laterally and dorsally at the midshaft. He was also suffering from Ureaplasma prostatitis. He admitted to use of anabolic steroids in the past, but not within three months of onset of symptoms. Duplex Doppler results were:- Rounded area of increased echo density to the right of the midline in the midshaft of the penis. Margins on $\mathbf{R}$ merge with that of the $\mathbf{R}$ corpus cavernosum: organised haematoma. Probably secondary to fracture $\mathrm{R}$ corpus cavernosum.

E. A 25 year old storeman presented on 28 May 1993 with a two week history of a lump in the penis and painful erections, following masturbation. No "crack" was heard. A $3 \mathrm{~cm}$ linear, cord-like lump was found, extending into a half circumferential lesion approximately $2 \mathrm{~cm}$ across and distal to the coronal sulcus. He also admitted to prior use of anabolic steroids. Duplex Doppler results were:- No flow in dorsal vein, reflecting presence of persisting thrombus. Compression with transducer failed to collapse the vein. Foci of calcification between corpora spongiosa mid-shaft. Focus calcification in midline ?old phleboliths from resolved thrombus. Antithrombin III was $123 \%$. Protein $S$ functional was $160 \%$. Final diagnosis: DVTP.

F. A 22 year old clerk presented on 3 March 1993 with gonorrhoea. On examination, he also had lymphoedema associated with distal penile swelling and a palpable thrombosed vein. Clinically a DVTP was confirmed. He declined further followup. All patients had normal INR, APTT, fibrinogen, protein $\mathrm{C}$ function and LCT ratio.

No common factors were found with regard to drug use, alcohol intake, smoking, intercurrent or past illness or sexual orientation. Three patients' family histories revealed type II diabetes mellitus in near relatives. There were no other relevant factors in family history. All full blood counts were within normal limits.

\section{Discussion}

A small tear in the tunica albuginea of subclinical nature can heal with subsequent scar formation. Impotence, fibrotic scarring and later penile deformity on erection have all been reported. ${ }^{1}$ The penile curvature may result in the diagnosis of Peyronie's disease being erroneously made. This happened to patient B in our series. Peyronie's is a systemic desmoplastic disease of unknown aetiology. ${ }^{2}$ It is possible that a substantial number of patients with penile deviation may actually have had some form of unremembered penile fracture causing insufficient symptoms to warrant presentation for medical attention. Thus, penile fracture is in the differential diagnosis of Peyronie's disease.

Penile fracture with extreme scarring and resultant severe curvature can result in secondary treatment being required, with insertion of a penile prosthesis to correct the deformity. ${ }^{8}$

Our patients presented between 7 and 14 days from onset of symptoms. Anselmo et al ${ }^{9}$ reported 13 patients who presented with fracture of the penis. All were operated on within 
the period 2 hours to 8 days after injury, with excellent functional results. Despite the favourable outcome, they emphasised the need for immediate surgery in penile fracture in order to prevent erectile failure and curvature.

DVTP, on the other hand, may be more common than RCC. ${ }^{10}$ The exact aetiology of the thrombus in DVTP is still in question. DVTP usually has few physical signs on visual inspection, but there is often a cordlike, linear, palpable mass in the region of the dorsal vein. This may be up to $5 \mathrm{~cm}$ long, whilst sometimes intermittent lumps are found with normal spaces between. Lymphoedema may confuse the issue. A history of insidious swelling appearing over 2 to 14 days strongly suggests the diagnosis.

Pure isolated DVTP does not cause erectile or ejaculatory problems and appears to resolve spontaneously. Isolated DVTP is probably best managed in a conservative manner. Early anticoagulants may be justifiable on theoretical grounds, but our followup results indicate that they are unnecessary. Anticoagulants in RCC (if misdiagnosed as DVTP) may worsen the situation and could cause excessive blood leakage, which could extend as far as the inguinal region. ${ }^{11}$

A unique case of disruption of the deep dorsal vein of the penis, with associated thrombus, was described in $1992 .{ }^{12}$ The authors comment on previous reports of dorsal artery rupture ${ }^{13}$ and rupture of the superficial dorsal vein ${ }^{14}$ as a result of sexual intercourse. A false negative cavernosagram after early sealing in the case of deep dorsal vein rupture supports the argument for surgical exploration. It is not known if the modality of penile doppler ultrasound was available to the authors concerned, ${ }^{12}$ but this could be a useful and non-invasive investigation where vascular disruption is clinically suspected.

There has been close agreement between

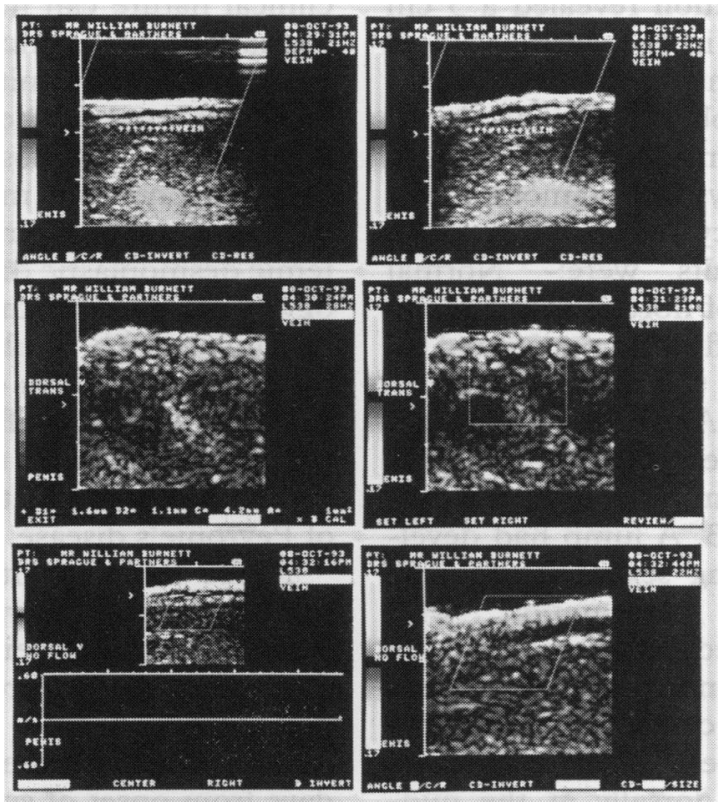

Figure Duplex Doppler ultrasound showing absence of flow in the dorsal vein. Areas of calcifications between the corpora spongiosa in mid-shaft are shown, that are probably old phleboliths. clinical assessment and followup of cases of DVTP and the Doppler findings. "Control" Doppler studies of dorsal vein bloodflow were done on routine patients sent in with vascular insufficiency or other penile problems. Dorsal vein flow was normal in virtually all cases. ItD follows, therefore, that the failure to demonstrate flow in the cases of this series is likely tof be truly significant (Personal Communication, Dr John Fraser, 1993). The fig-市 ure illustrates the definite appearance of absent bloodflow in the dorsal vein afteres DVTP.

Coagulation studies yielded interesting results, with elevated antithrombin III levels found in all patients. AT III is a major natura $\mathbb{B}^{\circ}$ inhibitor of activated coagulation factors and deficiency, rather than excess is to be expected in "unexplained" cases of thrombosis. ${ }^{15}$ AT III may be raised in infection, inflammation orw with use of anabolic steroids, but this does notes satisfactorily explain our findings. Two cases of mildly elevated protein $S$ levels are also seen in the patients with the highest AT III levels. Again, no satisfactory explanation cano be found for this; however the Doppler studies reveal them to be the patients withpersisting pure DVTP. Further inquiry intod underlying mild coagulation defects may be of use in elucidating the cause of DVTP.

Since the collection and investigation of the described case series, the authors have seen $\vec{x} \hat{0}$ further two cases of DVTP. In neither case ${ }^{\mp}$ could sexual trauma be convincingly implicated as a causative factor.

A new coagulation test, Activated Protein C Resistance, has recently become available iñ Western Australia. It is said to explain $50 \%$ op previously inexplicable thrombosis. ${ }^{16}$ This test will shortly be performed on blood samples from the study patients as well as the two new cases, if possible.

The normal physical findings and lack of significant past history suggest that there is nog known predisposing condition in the cases presented. Thus DVTP should not be and is not found to be a recurring condition. The authors suspect that DVTP is more likely if the penis comes out of the vagina/rectum durs ing coitus and the full weight of the partner is forced upon it at the base.

If DVTP is not considered as a differentiat diagnosis of the swollen and/or deformedw penis, much invasive investigation and patiente


ural history is benign, the authors caution thate historical evidence at least should be obtained with regard to conditions predisposing too thrombosis. Reassurance can then be readily, given that no major treatment is required $\mathbb{D}$ Doppler ultrasound may well become the्g investigation of choice in cases where diagnosis? is in doubt.

We thank Dr John Frazer for performing the Doppler examinations and Dr Ross Baker for the haematological testing. We also thank Mrs Doris Bhullar for her skill and patience with the initial typing.

1 Jordan GH, Gilbert DA. Male Genital Trauma. Genitourinary Surgery; Clinics in Plastic Surgery. 1988;15:431-2. 
2 Godec CJ, Raiser R, Logush AZ. The Erect Penis-Injury Prone Organ. $\mathcal{F}$ of Trauma 1988;28:124-6.

3 Creecy AA, Beazlie FS Jr. Fracture of the Penis; Traumatic Rupture of Corpora Cavernosa. $\mathcal{F}$ Urol 1957; 58:620.

4 Gallagher EP, Kiser WS. Injuries of the Corpua Cavernosum. F Urol 1961;85:949.

5 Kalash SS, Young JD Jr. Fracture of the Penis: Controversy of surgical versus conservative treatment. Urology 1984;24:21.

6 Throbeck RV, De la Maza in Za JM. Fracture de la Verge. $\mathcal{f}$ Chir (Paris) 1978;115:621.

7 Fernstrom U. Rupture of the Penis: Report on one operated case and review of the literature. Acta Chir Scand ated case and
$1957 ; 113: 211$

8 Ansell JS. The Crooked Penis: Causes and treatment. Medical Aspects of Human Sexuality 1991;32-6.

9 Anselmo G, Fandella A, Fassiano L, Merlo F, Macatrazzo L. Fractures of the Penis: Therapeutic approach and long-term results. Br F Urol 1991:67:509-11.

10 Tsang T, Demby AM. Penile Fracture with Urethral Injury. $₹$ Urol 1992;147:466-8.

11 Al Saleh BMS, Ansari ER, Al Ali IH, et al. Fractures of the Penis in Abu Dhabi. $\mathcal{f}$ Urol 1985;134:274-5.

12 Nicely ER, Costabile RA, Moul JW. Rupture of the Deep Dorsal Vein of the Penis During Sexual Intercourse. f Urol 1992;147:150-2.

13 Mostafa H. Rupture of the Dorsal Artery of the Penis as a result of Sexual Intercourse. $\mathcal{F}$ Urol 1967;97:314.

14 Waller DA, Britton JD, Ferro MA. Rotational Injury of the Penis. Br f Urol 1990;65:425.

15 McPherson J Thomas D (eds). Test Listing. In: Manual of Use and Interpretation of Pathology Tests. The Royal College of Pathologists of Australia, Sydney 1990; 136, College of Pathologists of Australia, Sydney 1990; 136,

16 Bauer KA. Hypercoagulability-A New Cofactor in the Protein C Anticoagulant Pathway. NEFM 1994;330: 566-7. 